HOST-MICROBE INTERACTIONS

DAO does it

Nat. Microbiol. 1, 16125 (2016)

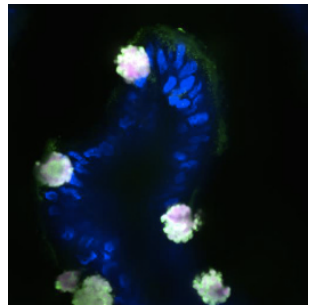

D-amino acids (D-aa) are not incorporated into proteins by ribosomes but function as signaling molecules, such as in mammalian neurotransmission, and as regulators of bacterial cell wall formation. Although bacteria have not been shown to produce D-aa previously, secretion of D-aa by commensal bacteria may affect host physiology. To test this, Sasabe et al. looked for D-aa and the D-aa-metabolizing enzyme DAO in the intestines of germ-free (GF) mice and mice free of only certain pathogens (SPF). The caeca of SPF mice expressed DAO and contained D-Ala, D-Asp, D-Glu and D-Pro, at levels much higher than in GF mice, which contained only relatively low levels of D-Asp. As well, DAO was expressed in the small intestine of SPF mice, secreted by enterocytes and goblet cells, and to a much lesser extent in GF mice. Lumenal, mucosal and intraepithelial concentrations of D-ala were higher in DAO-mutant mice. The authors also found that DAO is bactericidal against several enteric pathogens, including Vibrio cholerae, in vitro, through the production of $\mathrm{H}_{2} \mathrm{O}_{2}$ from oxidation of D-aa. Also, a microbiome analysis showed that the overall intestinal microbiota composition is altered in the absence of DAO. These results suggest that the microbiota influences the production of DAO in the intestine, playing a general role in protection of the mucosal surface.

\section{TRANSCRIPTIONAL REGULATION}

\section{Stuck in traffic Mol. Cell 63, 433-444 (2016)}

Kin28 is a kinase within the yeast TFIIH complex. Some evidence indicates a role for Kin28 in regulating transcription initiation and elongation through phosphorylation of the C-terminal domain of RNA polymerase II (Pol II), but reversible Kin28 inhibitor studies revealed minor effects on cellular viability and mRNA synthesis, suggesting that a low level of Kin 28 activity may be sufficient for transcriptional initiation. Rodriguez-Molina et al. proposed that irreversible/covalent inhibition might reduce Kin28 activity levels below this threshold and used a chemical-genetic approach to engineer mutations in the Kin28 ATPbinding pocket to enable binding by an ATP analog containing a thiol-reactive chloromethyl-ketone moiety (CMK). Yeast strains expressing this Kin28 irreversibleinhibitor-sensitized allele (kin28is) with CMK exhibited cellular lethality and greatly reduced serine phosphorylation in the C-terminal domain, resembling KIN28 genetic mutants. Transcriptome analysis of kin28is with CMK revealed low levels of nascent mRNA, and Pol II tracking
PROTEASOMES

\section{Attack of cancer drugs}

studies exhibited a buildup of Pol II at $5^{\prime}$ ends of protein-coding genes, revealing a role for Kin 28 in promoting transcriptional elongation. Consistent with a defect in elongation activity, combining kin28is with a fast-processing RNA polymerase variant rescued the growth defects. Altogether, these studies present a structure-guided approach to irreversibly sensitizing a desired kinase and underscore the power of the covalent inactivation strategy to resolve biological questions.

PHOSPHOLIPIDS

\section{How to flip a flippase}

Proc. NatI. Acad. Sci. USA 113, E4460-E4466 (2016)

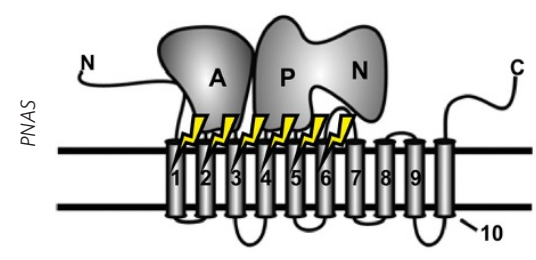

In eukaryotes, the asymmetry of the plasma membrane is critical to its proper function, and this asymmetry is maintained by phospholipid flippase enzymes that selectively transport phospholipids from the exofacial to the cytofacial leaflet of the membrane. In yeast, the P4-ATPase Dnf1 is a phosphatidylcholine (PC) flippase and does not transport sphingomyelin (SM), even though the two have the same choline headgroup and highly similar fatty acyl chains. To understand how Dnf1 exerts such selectivity for its phospholipid substrates, Roland et al. used a directed evolution library of Dnf1 variants in a screen with fluorescently labeled PC and SM to identify flippase sequences capable of selecting for SM instead of PC. A single amino acid change at the cytofacial side of the transmembrane domain enabled Dnf1 to select for the sphingosine backbone of SM over the glycerol backbone of PC. Homology modeling and additional mutagenesis further elucidated how this position, along with others nearby in the exit-gate region of the protein, discriminates between various phospholipid backbones as well as different headgroups. The insights into selectivity afforded by this study suggest how substrate phospholipids are coordinated by the exit gate and should enable the design of new substrate-specific flippases for future studies of membrane asymmetry. 